\title{
Location of Axillary nerve in relation to acromian process of Scapula: a Cadaveric study
}

\author{
Gosavi S. $\mathrm{N}^{1}$., Jadhav S. D ${ }^{2}$, Zambre B. $\mathrm{R}^{3}$, Vatsalaswamy P. ${ }^{4}$ \\ ${ }^{1,2,3}$ Dept. of Anatomy, PDVVPF's Medical College, Ahmednagar/MUHS/India \\ ${ }^{4}$ Dept. of Anatomy, Dr. D. Y. Patil Medical College/ D. Y. Patil/Pune, India
}

\begin{abstract}
Commonest cause of axillary nerve lesion is trauma. Many workers have studied the safe area for axillary nerve by measuring its distance from various landmarks. Sixty formalin fixed cadaveric shoulders were dissected. After proper exposure of axillary nerve and acromian process of scapula, distance of axillary nerve was measured from lateral edge, anterior and posterior edge of acromian process in neutral position. Distance was noted in adduction and abduction. Arm length and full arm length were measured. Mean, standard deviation, range was calculated. Mean distance of the nerve in neutral position was compared to the arm length and the full arm length using SPSS 11 software.

Mean distance from lateral edge of Acromian process was $5.62 \pm 0.6 \mathrm{~cm}$. In $90^{\circ}$ abduction it was 4.28 $\pm 0.6 \mathrm{~cm}$. and $5.75 \pm 0.6 \mathrm{~cm} .10^{\circ}$ adduction. In neutral position strong correlation was observed with arm length $(r=0.39, p<0.002)$ and with full arm length. $(r=0.29, p<0.02)$ Distance of axillary nerve depends on arm position and the nerve is more prone to injury in abduction. It showed stronger correlation with upper arm length. Deltoid incision of about $3.5 \mathrm{~cm}$ should theoretically prevent the injury to axillary nerve.
\end{abstract}

Key words: Acromian process, Axillary nerve, nerve injury, Shoulder surgery

\section{Introduction}

The commonest cause of axillary nerve lesion is trauma (dislocation of shoulder joint, fracture of the surgical neck of the humerus).[1] The fracture of proximal end of the humerus accounts for more than 5\% of all bone fractures and that such fractures largely occur among elderly osteoporotic patients. [2,3]

Many workers have studied the safe area for axillary nerve by measuring its distance from various landmarks like acromian process [4-6], head of the humerus [7], greater tubercle [8]and upper border of Deltoid [9] etc. The acromian process being an easily palpated bony landmark was used in the present study. The present study was carried out with an aim to study the distance of axillary nerve from acromian process of scapula and to correlate this distance with the arm length and full arm length. Secondly to note the effect of arm position on this distance. Knowledge of anatomy of the axillary nerve helps the surgeon in avoiding nerve injury during orhtroscopic procedures.

\section{Materials And Method}

Sixty formalin fixed cadaveric shoulders from Department of Anatomy were dissected. In each cadaveric shoulder, the acromian process of scapula was palpated, a vertical incision extending about six to eight $\mathrm{cm}$ was given, the fibers of deltoid were separated and the anterior branch of axillary nerve was identified at the surgical neck of the humerus. After proper exposure of the axillary nerve and the acromian process of scapula, the distance of the axillary nerve was measured from lateral edge, anterior and the posterior edge of the acromian process in neutral position. (arm by the side of the body) The distance was also noted in $10^{\circ}$ adduction and in $90^{\circ}$ abduction. The arm length was measured as the distance from the superior border of the lateral edge of the acromian process to the lateral epicondyle of the humerus. The Full arm length was measured as the distance from the superior border of the lateral edge of the acromian process to the radial styloid process, with the arm by the side of the body and extended at elbow joint.

The mean, standard deviation and range was calculated for these parameters. The mean nerve distance in neutral position was compared to the arm length and the full arm length using SPSS 11 software.

\section{Results}

After studying the position of axillary nerve in relation to anterior, posterior and lateral edge of the acromian process of scapula and also in various arm positions in sixty adult cadavers, the results were summarized in TABLE I. The mean distance from the lateral edge of Acromian process was $5.62 \pm 0.6 \mathrm{~cm}$, in $90^{\circ}$ abduction it was $4.28 \pm 0.6 \mathrm{~cm}$. The distance was slightly more in $10^{\circ}$ adduction ie. $5.75 \pm 0.6 \mathrm{~cm}$. In neutral position strong correlation was observed with the arm length $(\mathrm{r}=0.39, \mathrm{p}<0.002)$ and with the full arm length. $(\mathrm{r}$ $=0.29, \mathrm{p}<0.02)$ TABLE II. 


\section{Discussion}

The axillary nerve arises from the posterior cord $(\mathrm{C} 5,6)$. It traverses the quadrangular space and divides in the space into anterior and posterior branches. The anterior branch curves round the neck of the humerus with the posterior circumflex humoral vessels, deep to deltoid. It reaches the anterior border of the muscle and supplies it. The posterior branch frequently supplies the posterior aspect of the deltoid, usually via a separate branch from the main stem, occasionally from the superior lateral cutaneous nerve of the arm. However the posterior part of deltoid has more consistent supply from the anterior branch of the axillary nerve which should be remembered when performing the posterior deltoid splitting approach to the shoulder [1] After dissecting sixty cadaveric shoulders, the mean distance of axillary nerve from the lateral edge of the acromian process was $5.6 \mathrm{~cm}$ in the present study. Our findings were comparable with some of the previous studies. TABLE III

In the present study the mean distance of the nerve from the lateral edge of acromian process was minimum i.e. $4.28 \mathrm{~cm}$ during abduction and the finding was similar to Gulihar et al. [5] hence we are in agreement with Gulihar et al. [5] that, determination of safe distance between the lateral edge of the acromian and axillary nerve is particularly important with the arm in abduction. When the nerve is much closer to the acromian and it is at increased risk during surgery.

Gulihar et al. [5] calculated the safe distance using the mean minus three standard deviation, as this should theoretically prevent $99.7 \%$ of the nerve injury and in their study it was $4.2 \mathrm{~cm}$. After applying the same formula in the present study, we could determine the safe distance as $3.82 \mathrm{~cm}$.

The observations in the present study were also similar to Abbot et al, [4] who stated that, incision made with separation of the fibers of the deltoid should not extend downwards for more than 1.5 inch $(3.81 \mathrm{~cm})$ below the margin of the acromian.

The distance of the axillary nerve from acromian process is variable and can be correlated with the arm length, which is easy to measure during surgery. The arm length was observed as $32.71 \pm 2.6 \mathrm{~cm}$ in the present study and when correlated with the distance of axillary nerve from lateral edge of acromian process showed strong correlation. Strong correlation was also observed when the full arm length, ie $56.76 \pm 3.6 \mathrm{~cm}$, was correlated with the distance of axillary nerve.The association was stronger for arm length than for full arm length. These findings were similar to the observations made by Gulihar et al.[5]

Kontakis et al [9] measured the distance of Axillary nerve from the upper border of the deltoid muscle and correlated it with deltoid ratio. They found it as a useful guide for estimation of the position of the nerve at the time of surgery. We are in agreement with Gulihar et al. [5] that, measurement of the internal width and length of Deltoid is not a practical measurement for surgeons operating in shoulder region.

Cetik et al. [6] stated that, because the acromian can be described as a palpable line rather than a single point, for surgical dissection a safe area can be defined instead of a safe zone or a safe line. They described this as a quadrilateral area depending on the mean anterior and posterior distance of the nerve from the acromian process. They also found a strong correlation with the arm length. According to Cetik et al. [6] the average distance from the axillary nerve to the acromian was $6.08 \mathrm{~cm}$ and the minimum distance was $5.20 \mathrm{~cm}$. They suggested that to minimize the risk of axillary nerve damage, the shortest distance should be used during dissection. In the present study, the average distance of axillary nerve from acromian was $5.62 \mathrm{~cm}$ and the minimum distance was observed as $3.37 \mathrm{~cm}$. [only in two shoulders $(3.33 \%)$ the distance was less than $4 \mathrm{~cm}$ ] Hence the distance of $3.5 \mathrm{~cm}$ may help to avoid injury to axillary nerve during dissection (in majority).

\section{Conclusion}

The distance of axillary nerve is variable in its course. It depends on the arm position and the nerve is more prone to injury in abduction. The distance of the axillary nerve showed stronger correlation with the upper arm length than with the full arm length. The safe area for axillary nerve can be determined with the distance of the nerve from various points on the acromian process. The deltoid incision of about $3.5 \mathrm{~cm}$ should theoretically prevent the chances of injury to axillary nerve.

\section{Acknowledgement}

We are grateful to Dr. S. S. Dumbre Patil, consultant orthopedic surgeon, Noble hospital, Pune, for his valuable inputs for this article.

\section{References}

[1] Standring S, Gray's Anatomy- The anatomical basis of clinical practice $40^{\text {th }}$ edi. (London UK: Elsevier, 2008).

[2] Bigliani LU, Flatow EL, Pollock RG. Fracture of the proximal humerus. In: Rockwood CA, Matsen FA III, (Eds). The shoulder 2 nd ed. (Philadelphia: WB Saunders Co. 1998;1) 337 - 389.

[3] Lind T, Kroner K, Jensen J. The epidemiology of fractures of the proximal humerus. Arch Orthop Trauma Surg 108, 1989, 285 287.

[4] Abbott LC, deC JB, Saunders M, Hagey H, Jones EW Jr. Surgical approaches to the shoulder joint. J Bone Joint Surg Am 31, 1949, 235-5. 
Location of Axillary nerve in relation to acromian process of Scapula: a Cadaveric study

[5] Gulihar A, Sivaraman B. Matthew N, Taylor Grahame JS. A contribution to the calculation of a safe deltoid split. Int J Shoulder Surg 2(3 suppl), 2008, 52-5.

[6] Cetik O, Uslu M, Acar HI, Comert A, Tekdemir I, Cift H. Is there a safe area for Axillary nerve in the deltoid muscle? A cadaveric study. J Bone Joint Surg Am, 88, 2006, 2395-9.

[7] Traxler H, Surd R, Laminger KA, Windisch A, Sora MC, Firbas W. The treatment of subcapitate humoral fracture with dynamic helix wire and the risk of concomitant lesion of the Axillary nerve. Clinical anatomy, 14, 2001, 418-423.

[8] Boraiah S, Gardner MJ, Lorich DG. The surgical approach, its vascular implications and the importance of medial calcar support in maintaining fracture reduction in locked plating of proximal humerus fractures - A review. Pb Journal of Orthopedics vol-X No.1, $2008,14-20$

[9] Kontakis GM, Steriopoulos K, Damilakis J, Michalodimitrakis E. The position of Axillary nerve in the deltoid muscle: A cadaveric study. Acta orthop Scand, 70, 1999, 9-11

Table I - Observations of axillary nerve distance from the acromian process of scapula

\begin{tabular}{llcc}
\hline No & Parameter & Range $(\mathbf{c m})$ & Mean $\pm \mathbf{S D}(\mathbf{n}=\mathbf{6 0})(\mathbf{c m})$ \\
\hline & & & \\
1 & Distance From Anterior edge of Acromian & $3.85-6.11$ & $5.529 \pm 0.6$ \\
2 & Distance From Lateral edge of Acromian & $3.37-6.9$ & $5.627 \pm 0.6$ \\
3 & Distance From Posterior edge of Acromian & $4.27-6.74$ & $5.566 \pm 0.5$ \\
4 & In Abduction & $3.21-6.08$ & $4.281 \pm 0.6$ \\
5 & In Adduction & $4.02-7.21$ & $5.753 \pm 0.6$ \\
6 & Arm length & $29-36.79$ & $32.71 \pm 2.6$ \\
7 & Full arm length & $50-62.2$ & $56.76 \pm 3.6$ \\
\hline
\end{tabular}

Table II-Correlation of Distance of Axillary nerve from lateral edge of acromian process with arm length and full arm length

\begin{tabular}{llrc}
\hline No & Parameter & r value & P value \\
\hline & & & \\
1 & Arm length in Neutral position & 0.39 & $<0.002$ \\
2 & Full arm length in Neutral position & 0.29 & $<0.02$ \\
\hline
\end{tabular}

Table - III - Comparison with some of the previous studies

\begin{tabular}{lccc}
\hline Parameter & Present study & Gulihaer et al. ${ }^{(\mathbf{5})}$ & Cetik et al. $^{(\mathbf{6})}$ \\
\hline Anterior edge & $5.53 \pm 0.6$ & & $6.08 \pm 0.45$ \\
Lateral edge & $5.63 \pm 0.6$ & $6.0 \pm 0.5$ & \\
Posterior edge & $5.56 \pm 0.5$ & & \\
In Abduction & $4.28 \pm 0.6$ & $4.5 \pm 0.6$ & \\
In Adduction & $5.75 \pm 0.6$ & $6.2 \pm 0.5$ & \\
Arm length & $32.71 \pm 2.6$ & $31 \pm 2.2$ & $30.40 \pm 1.61$ \\
Full arm length & $56.76 \pm 3.6$ & $76.9 \pm 5.6$ & \\
\hline
\end{tabular}

